\title{
Su(var)3-9, Enhancer of Zeste, and Trithorax Domain-Containing 5 Facilitates Tumor Growth and Pulmonary Metastasis through Up-Regulation of AKT1 Signaling in Breast Cancer
}

\author{
Zhaoting Yang, ${ }^{* \dagger}$ Chengye Zhang, ${ }^{\dagger \dagger}$ Nan Che,${ }^{* \dagger}$ Ying Feng, ${ }^{* \dagger}$ Chao $\mathrm{Li}^{\dagger}{ }^{\dagger}$ and Yanhua Xuan ${ }^{* \dagger}$
}

From the Department of Pathology* and the Institute for Regenerative Medicine ${ }^{\dagger}$ Yanbian University Medicine College, Yanji; and the Air Force Medical Center of the Chinese People’s Liberation Army, ${ }^{\ddagger}$ Beijing, China

Accepted for publication

October 6, 2020.

Address correspondence to Yanhua Xuan, M.D., Ph.D., Department of Pathology, Yanbian University Medicine College, No. 977, Gongyuan Rd., Yanji 133002, China. E-mail: xuanyh1@ybu.edu.cn.

\begin{abstract}
Several studies have confirmed the function of Su(var)3-9, Enhancer of zeste, and Trithorax (SET) domaincontaining 5 (SETD5) in post-translational modifications of nonhistone proteins. Mutation of the SETD5 gene has been implicated in the progression of many human cancers, such as breast cancer (BC), but its functional role in $\mathrm{BC}$ progression is still unknown. The current article investigates the clinical significance and the functional role of SETD5 in BC. Our studies show that SETD5 expression in BC was related to poor clinical outcomes, including lymph node metastasis and advanced clinical stage. SETD5 expression positively correlated with tumor-associated macrophages. SETD5 was an independent predictor of poor overall survival in BC. Furthermore, these studies show that down-regulation of SETD5 significantly decreased BC cell proliferation, metastasis, and angiogenesis, and increased apoptosis of BC cells. The mechanistic analysis showed that SETD5 contributes BC progression by interacting with AKT1 pathway. Also, in vivo experiments show that blocking of SETD5 expression significantly inhibited tumor growth and pulmonary metastasis of BC cells. These findings indicate that SETD5 is a potential prognosis marker and facilitates tumor progression of BC. (Am J Pathol 2021, 191: 180-193; https://doi.org/10.1016/ j.ajpath.2020.10.005)
\end{abstract}

In 2018, approximately 2.1 million new breast cancer (BC) cases were diagnosed in females globally, accounting for almost one in four cancer cases in women. Current data show that $\mathrm{BC}$ is the most diagnosed cancer in most countries (154 of 185). Furthermore, it is the leading cause of cancer-related mortality in $>100$ countries. ${ }^{1}$ In 2019, the American Cancer Society's biennial update on female BC statistics in the United States indicated that over the most recent 5-year period, BC incidence rate has increased by $0.3 \%$ per year. $^{2}$ Despite success of hormonal and adjuvant chemotherapeutic agents to improve $\mathrm{BC}$ prognosis, treating the disease is still a challenge because it is still difficult to predict an individual's response to the treatment. Currently, efforts are being made to reduce the cost of treatment through the development of targeted therapies. As such, biomarkers have been effectively applied in BC diagnosis, prognosis, predicting response to therapy, as well as disease surveillance following treatment. ${ }^{3}$ Therefore, further elucidation of the underlying molecular mechanisms in $\mathrm{BC}$ progression is essential for the development of effective therapeutic agents, and the discovery of novel markers with have both prognostic and predictive value.

AKT, a serine/threonine kinase, is a signaling molecule of cell growth and differentiation. ${ }^{4}$ It is a well-characterized effector of phosphoinositide 3-kinase (PI3K) in PI3K/AKT/ mammalian target of rapamycin signaling pathway, and its deregulation plays a crucial role in the pathogenesis of many human cancers. ${ }^{4}$ Three AKT genes (AKT1, AKT2, and AKT3) have been identified in the mammalian genome, and

Supported by National Natural Science Foundation of China grant 81760531 (Y.X.).

Disclosures: None declared. 
the principal AKT isoform is encoded by AKT1, which modulates apoptosis. ${ }^{5}$ AKT1 is phosphorylated in vivo at Ser124, Thr308, Thr450, and Ser473. Moreover, the phosphorylation of Thr308 and Ser473 is generally inducible and mostly phosphorylated after treatment of cells with extracellular stimuli, whereas Ser124 and Thr450 appear to be basally phosphorylated. ${ }^{6}$ Genetic and epigenetic changes in genes participating in the AKT pathway activate AKT in human cancers. ${ }^{7}$ Although the direct effect of methylation on AKT activation is yet to be reported, methylation of its upstream regulators in many cancers was shown to activate AKT. ${ }^{8}$

Su(var)3-9, Enhancer of zeste, and Trithorax (SET) domain-containing 5 (SETD5) is a member of the histone lysine methyltransferase family that is yet to be characterized and typically possesses conserved SET domain. ${ }^{9}$ The enzyme catalyzes monomethylation, dimethylation, or trimethylation of lysine $\varepsilon$-amine groups of proteins, specifically histones, which facilitates rearrangement of chromatins and regulation of gene expression. Epigenetic regulation appears to contribute to the initiation and progression of cancer and may precede genetic mutations. Dillon et $\mathrm{al}^{10}$ assumed that SETD5 protein participates in epigenetic regulation. SETD5 gene mutations are associated with human neurodevelopmental disorders. ${ }^{11-14}$ Liu et a ${ }^{15}$ reported that mRNA levels of SETD5 were up-regulated in BC and significantly associated with shorter survival in patients, but the functional role of SETD5 in $\mathrm{BC}$ progression is not fully understood.

This article investigates the clinical significance and the functional role of SETD5 in BC. Our studies show that expression of SETD5 predicts poor prognosis of BC. We hypothesize that SETD5 contributes BC progression through activation of AKT1 signaling. Of importance, our data indicate that SETD5 facilitates BC tumor growth and pulmonary metastasis and could be a potential prognosis marker.

\section{Materials and Methods}

\section{Tissue Specimens}

Tissue microarray used for immunohistochemistry (IHC) staining was purchased from Shanghai Outdo Biotech Co, Ltd (Shanghai, China). This study was conducted as per the Declaration of Helsinki principles and was approved by the Human Ethics Committee and the Research Ethics Committee of Yanbian University (Yanji, China). Tissue microarray contained $117 \mathrm{BC}$ tissues that were previously fixed in formalin and then embedded in paraffin. The median follow-up duration was 112 months (range, 2 to 136 months).

\section{Cell Culture, Reagents, and Transfection}

MCF-7, MDA-MB-231, and human umbilical vein endothelial cells (ATCC, Manassas, VA) were grown under conditions specified by the supplier. All cell lines were Mycoplasma free. The following reagents were purchased for in vitro experiments: LY294002 (MedChem Express, Shanghai, China) and perifosine (Selleckchem, Shanghai, China). Negative control and SETD5 endoribonuclease-prepared short interfering RNA (esi-SETD5) were transfected into cells with Lipofectamine 3000 (Invitrogen, Carlsbad, CA) as per the methods described by the manufacturer. siRNA-universal negative control and esi-SETD5 were purchased from Sigma-Aldrich (St. Louis, MO). Table 1 lists the sequence of esi-SETD5.

\section{IHC, Western Blot, Immunofluorescence, and Migration and Invasion Assays}

IHC staining, ${ }^{16}$ Western blot, ${ }^{17}$ immunofluorescence, ${ }^{18}$ and migration and invasion assays ${ }^{18}$ were conducted according to previous protocols. Table 2 provides a list of antibodies used. $\beta$-Actin served as control. All experiments were repeated at least three times with similar results each time.

\section{Semiquantitative Analysis of Immunostaining}

The staining intensity and the proportion of positive cells were measured by pathologists (Y.X. and Z.Y.) with no prior knowledge of clinicopathologic results, and then staining score was assigned as follows: IHC score 1, weak staining in $<50 \%$ or moderate staining in $<20 \%$ of cancer cells; IHC score 2 , weak staining in $\geq 50 \%$, moderate staining in $20 \%$ to $50 \%$, or strong staining in $<20 \%$ of cancer cells; and IHC score 3 , moderate staining in $\geq 50 \%$ or strong staining in $\geq 20 \%$ of cancer cells. After having assessed IHC score, positivity of each case was decided according to cutoff point derived from clinical study, taking clinical end points such as recurrence and patients' survival. In this study, cases with IHC score 2 and 3 were regarded as positivity for SETD5 protein expression.

\section{Colony Formation Assay}

In brief, cells were plated (200 cells/well) on 6-well plates and then maintained for 2 weeks. Next, the colonies were fixed with paraformaldehyde and stained with crystal violet (1\%). Subsequently, the colonies were imaged to determine the number of colonies ( $>50$ cells/colony). Data were graphed as means \pm SD of experiments performed three times, each in triplicate.

\section{Wound-Healing Assay}

Cells were seeded in a 6-well plate and cultured for 24 hours to form confluent monolayers. A wound was generated by dragging a pipette tip through the monolayer, and plates were washed using prewarmed phosphate-buffered saline to remove cellular debris. Cell migration was monitored for 0 and 24 hours, and images were captured at each time point using a digital camera attached to an inverted phase contrast microscope. Data were graphed as means \pm SD for experiments performed three times, each in triplicate. 
Table 1 The Sequence of SETD5 Endoribonuclease-Prepared Short Interfering RNA

\begin{tabular}{ll}
\hline Gene & Sequence \\
\hline SETD5 & 5'-GATGTACAGAACGCGCTTGAACAACACCTACATTCTAGCAAGGAATTTGTGGGCAAACCTACTATTTTAGACACTATTAA \\
TAAGACTGAATTGGCCTGTAATAACACAGTTATTGGTTCCCAAATGCAGTTACAGCTGGGAAGAGTCACTCGTGTTCAAAA & GCACCGGAAGATCTGAGGGCTGCAAGAGATTTGGCTTGGACACTCTTATAATAGAGTATCGTGGGAAAGTCATGTTACG \\
ACAGCAATTTGAGGTCAATGGGCATTTCTTCAAAAAACCATACCCCTTTGTGCTCTTCTACTCAAAATTCAATGGTGTAGA \\
GATGTGTGTGGATGCCCGTACTTTCGGTAATGATGCTCGGTTCATCAGAAGATCATGTACACCAAATGCAGAGGTGCGACA \\
CATGATTGCAGATGGG-3' \\
\hline
\end{tabular}

\section{Cell Cycle Assay}

After being transfected with esi-SETD5 for 24 hours, the cells were fixed at $4^{\circ} \mathrm{C}$ in ethanol $(70 \% \mathrm{v} / \mathrm{v})$ in phosphate-buffered saline for 24 hours. After that, they were stained for 30 minutes with propidium iodide solution $(10 \mu \mathrm{g} / \mathrm{mL})$ comprising $5 \mu \mathrm{g} /$ $\mathrm{mL}$ RNase A. Flow cytometry with fluorescence-activated cell sorting was used to examine the cell cycle progression. All experiments were repeated at least three times.

\section{Carboxy-Fluorescein Succinimidyl Ester Staining}

The BC cells $\left(1 \times 10^{6}\right.$ cells $\left./ \mathrm{mL}\right)$ were suspended in RPMI 1640 medium, then labeled at $37^{\circ} \mathrm{C}$ for 20 minutes using 5 $\mu \mathrm{mol} / \mathrm{L}$ carboxy-fluorescein succinimidyl ester (Invitrogen). To quench the labeling reaction, RPMI 1640 medium containing fetal bovine serum $(10 \%)$ was added, and the mixture was incubated for 10 minutes. The carboxyfluorescein succinimidyl ester-labeled cells were washed

Table 2 Antibodies in This Study

\begin{tabular}{lll}
\hline Antibodies against & Company & Catalog number \\
\hline SETD5 & NOVUS (Centennial, C0) & NBP2-38257 \\
PI3K & Abcam (Cambridge, UK) & Ab86714 \\
p85 $\alpha$ & Abcam & ab86714 \\
AKT1 & Invitrogen & PA5-29169 \\
pAKT-Ser473 & Millipore (Billerica, MA) & $05-1003$ \\
pAKT-Thr308 & Millipore & $07-1398$ \\
Snail & Abcam & ab53519 \\
E-cadherin & Abcam & ab40772 \\
Vimentin & Millipore & MABT121 \\
Twist1 & Abcam & Ab50887 \\
$\beta$-Catenin & Invitrogen & TA2-62560 \\
Bcl-2 & Santa Cruz Biotechnology & Sc-7382 \\
& (Dallas, TX) & \\
Bax & Santa Cruz Biotechnology & Sc-493 \\
Caspase-8 & Abcam & Ab25901 \\
Cleaved caspase-3 & CST (Danvers, MA) & 5 A1E \\
Cleaved PRAP & Abcam & Ab32064 \\
VEGF & Millipore & ABS82 \\
EP0 & Abcam & Ab226956 \\
CD105 & Abcam & Ab170943 \\
Ki-67 & Abcam & Ab92742 \\
D2-40 & Proteintech (Wuhan, & 1 D9F3 \\
& China) & \\
\hline
\end{tabular}

Bax, Bcl2 associated X; Bcl-2, B-cell lymphoma 2; EPO, erythropoietin; pAKT, phosphorylated AKT; PI3K, phosphoinositide 3-kinase; PRAP, poly ADP ribose polymerase; VEGF, vascular endothelial growth factor. twice with culture medium and then seeded onto 6-well plates. The cells were harvested at the same time. The rate of cell proliferation was examined using C6 flow cytometry. All values are means of three experiments. Statistical analysis experimental values are presented as the means $\pm \mathrm{SD}$ for three independent experiments.

\section{Hoechst 33258 Staining}

Hoechst 33258 staining was conducted using Hoechst Staining Kit (Beyotime Biotechnology, Shanghai, China) to detect the nuclei morphology, as per the methods described by the manufacturer. The experiments were biologically repeated for three times, and data are shown as means \pm SD.

\section{Co-IP Data}

The Thermo Scientific Pierce co-IP kit (Thermo Scientific, Rockford, IL) was used to perform co-immunoprecipitation (co-IP) following the instructions provided by the manufacturer. Results shown are representative of experiments performed at least three times with similar results each time.

\section{Tube Formation Assay}

Briefly, cold Matrigel (Corning Inc., Corning, NY) was used to coat the 96-well plate. Next, the plates were incubated at $37^{\circ} \mathrm{C}$ for 4 hours, and then human umbilical vein endothelial cells $\left(2 \times 10^{4}\right.$ cells/well $)$ in $150 \mu \mathrm{L}$ conditioned medium, diluted at 2:1 in RPMI 1640 medium without fetal bovine serum, were seeded to Matrigel-coated wells and incubated for 4 to 6 hours at $37^{\circ} \mathrm{C}$. A microscope was used to observe the formation of capillary-like structures. Image $\mathrm{J}$ software version $1.52(\mathrm{NIH}$, Bethesda, MD; http://imagej. en.softonic.com, last accessed October, 2019) was used to assess total tube length and branching points formed by endothelial cells per image field. Here and later, data were graphed as means \pm SD for experiments performed three times, each in triplicate.

\section{Vasculogenic Mimicry Assay}

A total of $3 \times 10^{4}$ cells in RPMI 1640 medium without fetal bovine serum were seeded onto 96-well plates precoated with Matrigel. Tube formation was assessed under a phasecontrast microscope for 4 to 6 hours after seeding. 


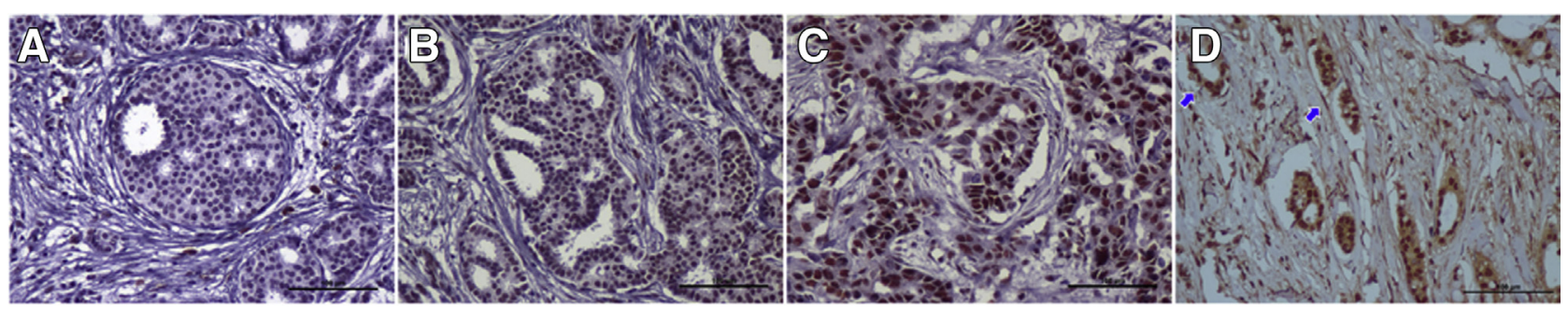

$\mathbf{E}$

Protein expression of SETD5 in breast cancer

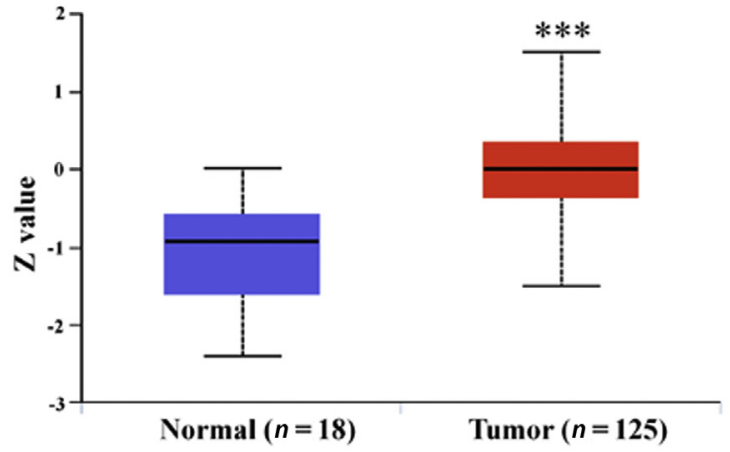

$\mathbf{F}$

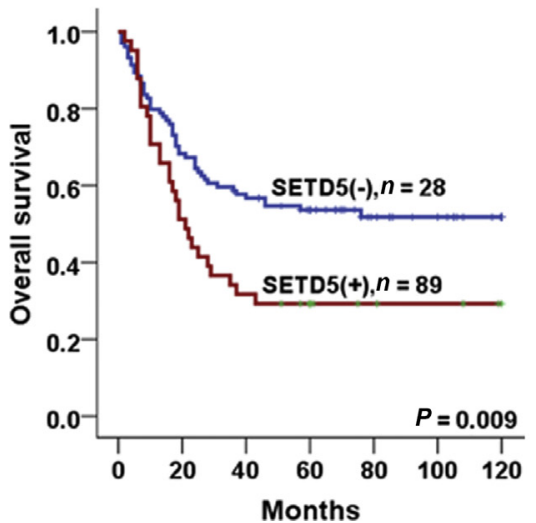

Figure 1 SETD5 expression is related to poor prognosis in breast cancer (BC). Immunohistochemistry on tissue microarrays for SETD5 to investigate SETD5 expression in human BC. A-C: SETD5 expressed in ductal carcinoma in situ tissues (A) and invasive ductal carcinoma of breast (B and C). D: Immunostaining of SETD5 (red reaction product) in the cancer cells and D2-40 (brown reaction product) around the cancer cells in the lymphatic invasion of breast ductal carcinoma (blue arrows). E: SETD5 protein expression detected in normal tissues and BC tissues using the online web portal UALCAN (http://ualcan.path.uab.edu, last accessed July, 2020) based on Clinical Proteomic Tumor Analysis Consortium. F: Kaplan-Meier analysis employed to study the relationship between SETD5 expression and overall survival (OS) in patients with BC. OS outcomes of the SETD5-positive group of patients (solid red line) versus the SETD5-negative group of patients (solid blue line). The OS time in months is displayed on the $x$ axis, and the probability of OS is displayed on the $y$ axis. $n=18$ normal tissues $(\mathbf{E}) ; n=125 \mathrm{BC}$ tissues $(\mathbf{E}) .{ }^{* * *} P<0.001$ versus controls (log-rank test). Scale bars $=100 \mu \mathrm{m}(\mathbf{A}-\mathbf{D})$. Original magnification, $\times 200($ A-D) .

\section{GSEA Data}

The raw data can be accessed in the National Center for Biotechnology Information Gene Expression Omnibus site (https://www.ncbi.nlm.nih.gov/geo; accession number GSE29431, last accessed May, 2019). The database was analyzed using the Gene Set Enrichment Analysis (GSEA). GSEA version 2.2.4 software (http://www. broadinstitute.org/gsea, last accessed May, 2019) was used to explore the relationship between SETD5 expression and PI3K/AKT signaling. The GSEA includes three key statistics: false discovery rate, nominal $P$ value, and normalized enrichment score.

\section{In Vivo Experiments}

All animal experiments were conducted as per the recommendations of the Guide for the Care and Use of Laboratory Animals ${ }^{19}$ of the NIH and were approved by the Yanbian University Animal Care and Use Committee. A total of $100 \mu \mathrm{L}$ of cell suspension MDA-MB-231 cells $\left(1 \times 10^{7}\right)$ in phosphate-buffered saline comprising $50 \%$ Matrigel was inoculated (subcutaneously) into the right or left flank of 5-week-old BALB/c nude female mice (Vital Rivers, Beijing, China) to generate a tumor model. Tumors were harvested at 4 weeks for cells after inoculation. To detect lung metastasis, MDA-MB-231 cells $\left(1 \times 10^{6}\right)$ in saline were injected into the tail vein of nude mice. The mice were sacrificed at 4 weeks following injection. The nodules were counted after harvesting the lungs. For histologic examination, hematoxylin and eosin staining was conducted.

\section{Statistical Analysis}

Data analysis was performed using SPSS version 25.0 (SPSS, Inc., Chicago, IL) and GraphPad Prism version 5.01 (GraphPad Inc., San Diego, CA). Correlation analysis was performed using Spearman correlation analysis. Overall survival (OS) was calculated using the Kaplan-Meier method followed by the log-rank test (for comparing the survival curves). Survival time was the period from the date of surgery to the date a patient died or the date of the last follow-up. The Cox proportional hazards model was used for multivariate analysis. Clinicopathologic factors, which were statistically significant in univariate analysis, were 
Table 3 Comparison of Clinicopathologic Characteristics According to the SETD5 Expression in Breast Cancer Tissues

\begin{tabular}{|c|c|c|c|c|c|c|}
\hline Variable & $n$ & SETD5 $(-), n(\%)$ & SETD5 $(+), n(\%)$ & $\chi^{2}$ & $\mathrm{R}$ & $P$ value \\
\hline Age, years & & & & 5.922 & 0.237 & 0.015 \\
\hline$\leq 50$ & 82 & $25(30.5)$ & $57(69.5)$ & & & \\
\hline Grade & & & & 0.649 & 0.012 & 0.723 \\
\hline Well & 31 & $9(29.0)$ & $22(71.0)$ & & & \\
\hline Moderately & 43 & $9(20.9)$ & $34(79.1)$ & & & \\
\hline $\mathrm{T} 1$ & 10 & $3(30.0)$ & $7(70.0)$ & & & \\
\hline $\mathrm{T} 2$ & 50 & $15(30.0)$ & $35(70.0)$ & & & \\
\hline T3 & 48 & $10(20.8)$ & $38(79.2)$ & & & \\
\hline $\mathrm{T} 4$ & 9 & $0(0.0)$ & $9(100.0)$ & & & \\
\hline Lymph node metastasis & & & & 15.786 & 0.388 & $<0.001$ \\
\hline II & 35 & $8(22.9)$ & $27(77.1)$ & & & \\
\hline III & 29 & $2(6.9)$ & $27(93.1)$ & & & \\
\hline IV & 7 & $0(0.0)$ & $7(100.0)$ & & & \\
\hline ER & & & & 1.885 & 0.127 & 0.170 \\
\hline Negative & 62 & $18(29.0)$ & $44(71.0)$ & & & \\
\hline Positive & 55 & $10(18.2)$ & $45(81.8)$ & & & \\
\hline PR & & & & 1.270 & 0.104 & 0.260 \\
\hline Negative & 56 & $16(28.6)$ & $40(71.4)$ & & & \\
\hline Positive & 61 & $12(19.7)$ & $49(80.3)$ & & & \\
\hline HER2 & & & & 1.631 & 0.118 & 0.202 \\
\hline Negative & 41 & 7 (17.1) & $34(82.9)$ & & & \\
\hline
\end{tabular}

ER, estrogen receptor; HER2, human epidermal growth factor receptor 2; PR, progesterone receptor; pT, pathologic tumor.

included as covariables in multivariate analysis. Hazard ratio and 95\% CIs were assessed for each factor. Two-group comparisons were analyzed using $t$-test (two tailed). $P<0.05$ was considered statistically significant.

\section{Results}

\section{Expression of SETD5 Is Related to Unfavorable Clinicopathologic Characteristics and Poor Disease Outcomes in BC}

Genetic mutation in the gene (SETD5) that codes for histone lysine methyltransferases has been shown to be significantly related to the progression of $\mathrm{BC}$ and OS of patients. ${ }^{15}$ As a methyltransferase, SETD5 controls histone acetylation, mediates transcription, and functions in cell cycle progression and embryo development in mammals. ${ }^{20}$ To fully explore the clinical significance of SETD5 in the context of BC, tissue microarray analysis was performed on 117 primary human BC specimens which were subsequently stained for SETD5 expression using IHC (Figure 1, A-D). The current results indicate that $89 \mathrm{BC}$ cases $(76 \%)$ were positive for SETD5 staining (Table 3). SETD5 had a low expression in ductal carcinoma in situ tissues (pT0) (Figure 1A), and medium expression in invasive ductal carcinoma of breast (pT1 and pT3) (Figure 1, B and C). The high expression of SETD5 cancer cells and D2-40-positive lymph vessels was observed in the lymphatic invasion of breast ductal carcinoma samples (Figure 1D). Among 51 cases of patients with lymph node metastasis, 48 (94\%) were positive for SETD5 staining, which was significantly higher compared with those for patients without lymph node metastasis $(62.1 \%)(P<0.001)$ (Table 3). The prevalence of SETD5 expression gradually increased with disease progression. In patients with $\mathrm{BC}$ with early clinical stage disease, $60.9 \%$ exhibited positive staining for SETD5, whereas in patients with more advanced stage II, stage III, and stage IV clinical disease, the prevalence was $77.1 \%, 93.1 \%$, and $100 \%$, respectively $(P<0.001)$ (Table 3). The association between SETD5 protein expression and clinical pathologic diagnosis from Clinical Proteomic Tumor Analysis Consortium database in BC tissues was also analyzed. The results obtained from the online tool 
Table 4 Univariate and Multivariate Analysis for Prognostic Variables of Overall Survival in Breast Cancer Patients Using Cox Proportional-Hazards Regression

\begin{tabular}{|c|c|c|c|c|c|c|}
\hline \multirow[b]{2}{*}{ Characteristic } & \multicolumn{3}{|c|}{ Univariate analyses } & \multicolumn{3}{|c|}{ Multivariate analyses } \\
\hline & $H R$ & $95 \% \mathrm{CI}$ & $P$ value & HR & $95 \% \mathrm{CI}$ & $P$ value \\
\hline Age, years & & & 0.097 & & & 0.088 \\
\hline$\geq 50$ & 1.493 & $0.931-2.397$ & & 1.627 & $0.931-2.842$ & \\
\hline pT stage & & & 0.001 & & & 0.009 \\
\hline $\mathrm{T} 1$ & 1.00 & & & 1.00 & & \\
\hline $\mathrm{T} 4$ & 8.307 & $2.315-29.817$ & & 7.141 & $1.422-35.858$ & \\
\hline Lymph node metastasis & & & 0.002 & & & 0.164 \\
\hline Negative & 1.00 & & & 1.00 & & \\
\hline Positive & 2.130 & $1.326-3.422$ & & 1.512 & $0.845-2.706$ & \\
\hline Distant metastasis & & & 0.012 & & & 0.110 \\
\hline Positive & 1.830 & $1.153-2.904$ & & 1.687 & $1.034-2.754$ & \\
\hline
\end{tabular}

HR, hazard ratio; $\mathrm{pT}$, pathologic tumor.

UALCAN showed that SETD5 protein expression significantly increased in $\mathrm{BC}$ tissues relative to that in the normal tissues $(P<0.001)$ (Figure 1E). Kaplan-Meier analysis showed that SETD5 expression in BC specimens was significantly associated with poor OS of patients $(P=0.009)$ (Figure $1 \mathrm{~F}$ ). Univariate Cox regression analysis identified pathologic tumor stage $(P=0.001)$, lymph node metastasis $(P=0.002)$, distant metastasis $(P=0.012)$, and SETD5 expression $(P=0.010)$ as significant prognostic factors of poor OS for patients with BC (Table 4). Multivariate Cox regression analysis revealed that pathologic tumor stage $(P=0.009)$ and SETD5 expression $(P=0.036)$ were independent prognostic factors for poor OS (Table 4). These findings imply that SETD5 expression may be a potential biomarker for poor prognosis of $\mathrm{BC}$.

\section{SETD5 Expression Positively Correlates with TAMs in BC}

Properties of tumor-associated macrophages (TAMs) that help in the progression of the tumor, their main functional peculiarities related to the suppression of immune reaction, extracellular matrix remodeling, and angiogenesis stimulation remain to be the most important factors for tumor development. $^{21}$ SETD5 had high expression in adjacent macrophage population of $\mathrm{BC}$, implying TAM population infiltration along with high SETD5 expression in areas around the cancer cells (Figure 2A). SETD5 expression positively correlated with TAM makers, such as CD68 $(P<0.001)$, CD204 $(P<0.001)$, CD206 $(P<0.001)$, T-cell immunoglobulin mucin family member 3 $(P<0.001)$, and CD163 $(P=0.0048)$ in BC samples by Gene Expression Profiling Interactive Analysis through The
Cancer Genome Atlas database (Figure 2, B-F). Thus, TAMs with high SETD5 expression play an important role in progression of BC. However, further studies are required to determine a specific relationship between SETD5 expression and TAM infiltration in BC.

\section{Inhibition of SETD5 Expression Suppresses BC Cell Proliferation}

SETD5 was not related to progesterone receptor, estrogen receptor, or human epidermal growth factor receptor 2 (Table 3). Thus, aggressive triple-negative MDA-MB-231 and nonaggressive estrogen receptor $\alpha$-positive MCF-7 cell lines were used as a contrast in BC. To determine how SETD5 affects BC progression, the impact of SETD5 on cell apoptosis and proliferation was examined. First, SETD5 expression in MDA-MB-231 and MCF-7 cells was inhibited by transfecting them with SETD5-targeted endoribonuclease-prepared short interfering RNA (Figure 3A). Hoechst 33258 staining revealed significant increases in the morphologic hallmarks of apoptotic cell death in SETD5 knockdown cells relative to the control cells (Figure 3B). These observations were confirmed through the Western blot assay. The results of the current study indicates that inhibition of SETD5 expression significantly enhances the expression of apoptotic markers in BC cells, including caspase-8, cleaved poly ADP ribose polymerase, cleaved caspase-3, and $\mathrm{Bcl} 2$ associated X/B-cell lymphoma 2 (Figure 3C). In addition, carboxy-fluorescein succinimidyl ester assays (Figure 3D) and colony formation assays (Figure 3E) indicated that down-regulation of SETD5 significantly inhibited cell clonogenicity $(P<0.001)$ and 
A

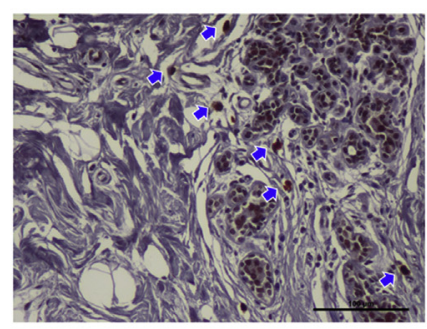

B

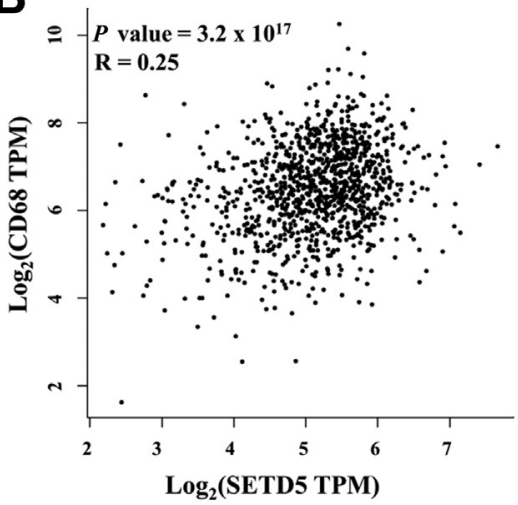

$\mathbf{E}$

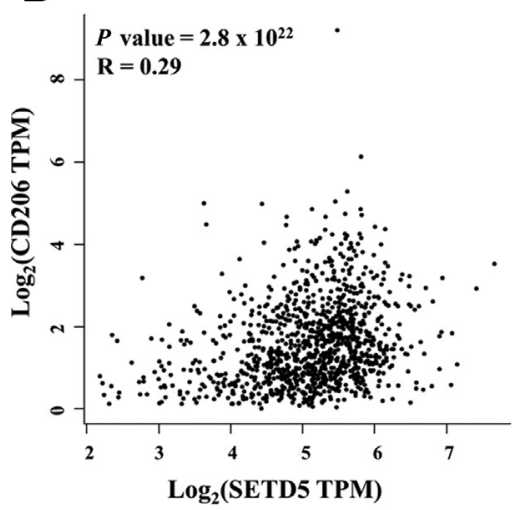

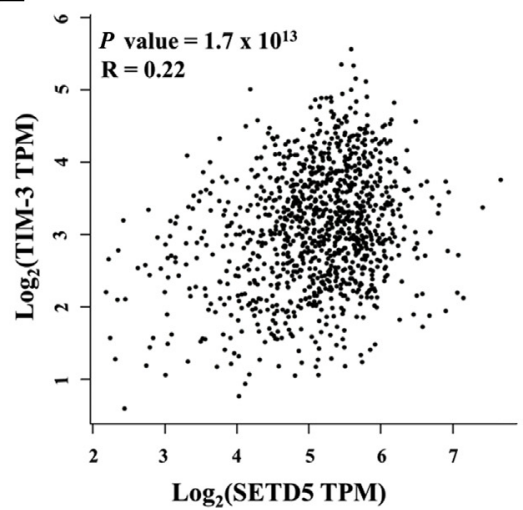

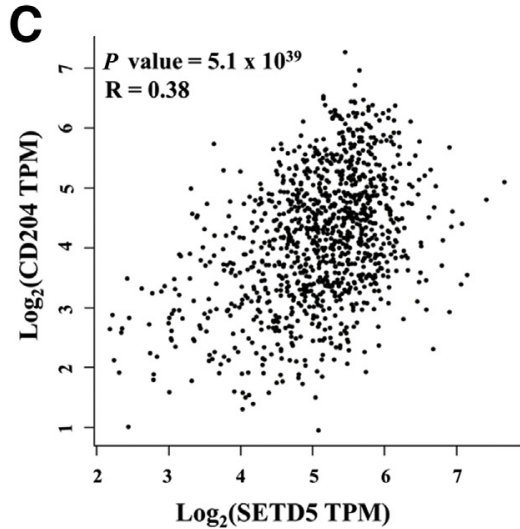

$\mathbf{F}$

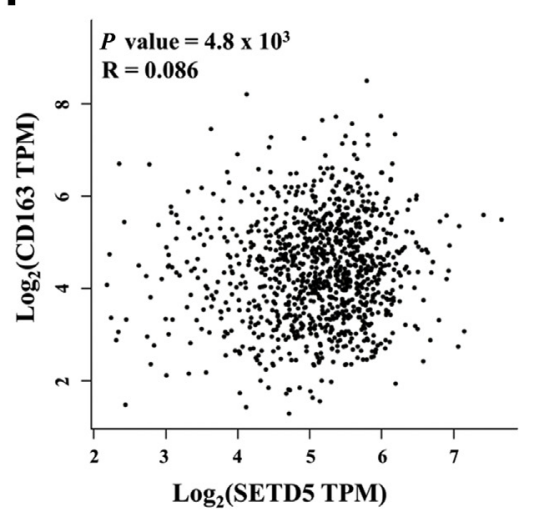

Figure 2 SETD5 expression positively correlates with tumor-associated macrophages (TAMs) in breast cancer (BC). A: Immunohistochemistry on tissue microarrays for SETD5 to investigate SETD5 expression in adjacent macrophage of breast ductal carcinoma cells (blue arrows). B-F: The correlation between SETD5 and mRNA levels of TAM makers CD68 (B), CD204 (C), CD206 (D), T-cell immunoglobulin mucin family member 3 (TIM-3; E), and CD163 (F) was analyzed in BC tissues by Gene Expression Profiling Interactive Analysis ( $h$ ttp://gepia.cancer-pku.cn, last accessed July 20, 2020). Scale bar $=100 \mu \mathrm{m}$ (A). Original magnification, $\times 200(\mathbf{A})$. TPM, transcript per million.

proliferation $(P<0.001)$. Flow cytometry analyses showed that knockdown of SETD5 expression primarily decreased the subpopulations in MDA-MB-231 and MCF-7 cells in S phase (Figure 3F). Collectively, these results indicate that SETD5 contributed to BC progression.

\section{SETD5 Expression Is Present in Conjunction with EMT-Like Changes in $\mathrm{BC}$}

Tumor budding is a phrase used to describe a thin anaplastic cell cord, undifferentiated tumor cells, and individual free cells at the invasion front of tumors and is recognized as being composed of neoplastic epithelium or cancer cell aggregates (up to five cells). ${ }^{22}$ SETD5 expression was diffuse yet strong in the tumor budding of BC specimens (Supplemental Figure S1). Tumor budding is closely related to epithelial-mesenchymal transition (EMT), and the cells exhibit both invasive and migratory characteristics. ${ }^{23,24}$ EMT is an important biological process that involves the release of epithelial cells from the surrounding tissue, which then gain the features of mesenchymal cells, such as distinctive, motile, spindle-shaped cells with end-to-end polarity. Therefore, the effects of inhibiting SETD5 expression on EMT were assessed. Frequently used molecular indicators of EMT include up-regulation of vimentin and $\mathrm{N}$-cadherin and increased production of transcription factors that inhibit E-cadherin and $\beta$-catenin production, including Slug, Snail, Twist, and zinc finger E-box binding homeobox $1 .^{25,26}$ These studies showed that inhibiting SETD5 expression significantly decreased the levels of Snail, Twist1, and vimentin proteins and significantly increased the levels of E-cadherin and $\beta$-catenin proteins in MCF-7 and MDA-MB-231 cells (Figure 4A). Immunofluorescence staining further confirmed that esi-SETD5 up-regulated E-cadherin, while downregulating vimentin (Figure 4B). EMT plays an essential role during the initial stages of metastasis. ${ }^{25}$ To investigate the function of SETD5 in metastasis of BC cells, transwell and wound-healing assays were performed. Data from these assays indicate that SETD5 silencing markedly suppressed the invasive and proliferative abilities of BC cells (Figure 4C and Supplemental Figure S2). Collectively, these findings indicate that SETD5 facilitates the migration and invasion of cells during $\mathrm{BC}$ progression via EMT.

\section{SETD5 Induces Tumor Angiogenesis}

The clinical failure of anti-angiogenic drugs in the treatment of $\mathrm{BC}$ may be attributed to the fact that many factors 
A

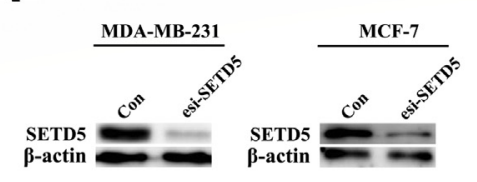

B

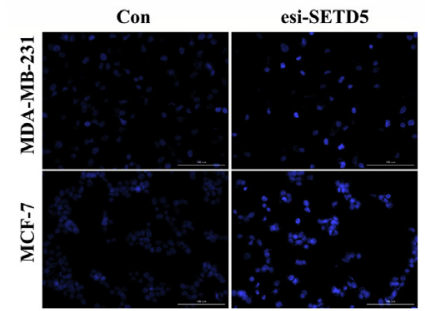

C
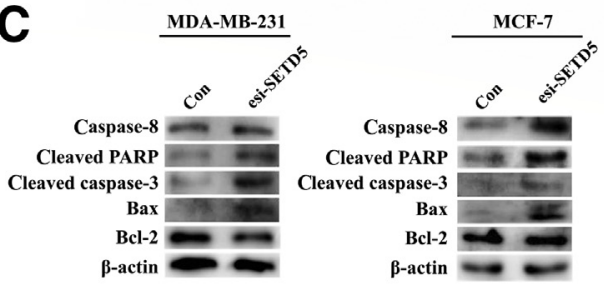

$\mathbf{E}$
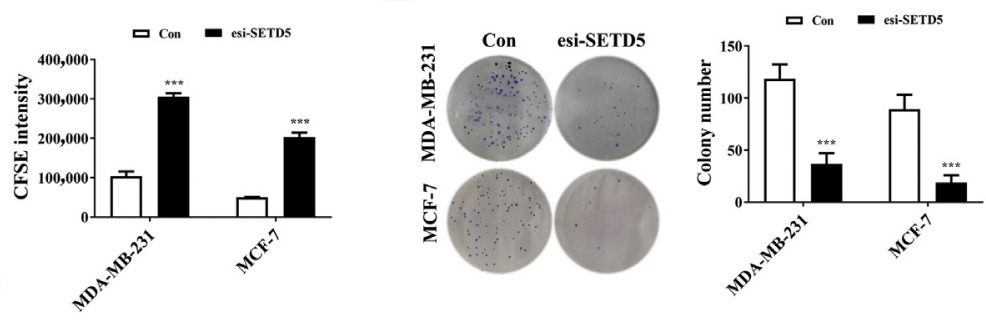
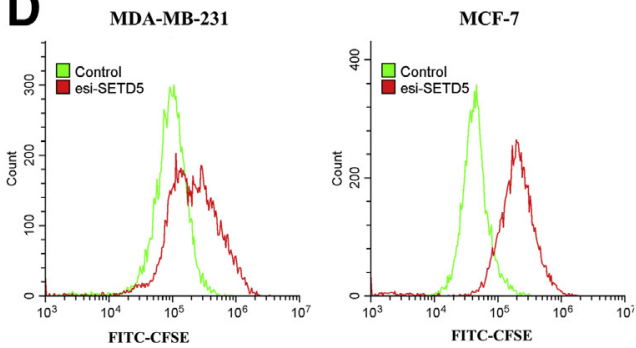

F

MDA-MB-231

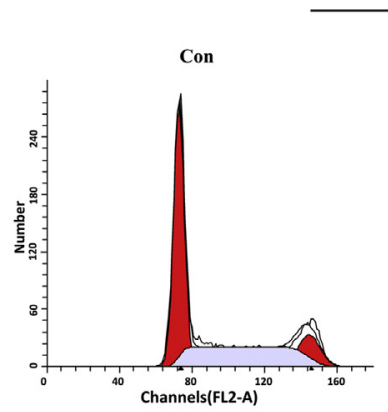

MCF-7
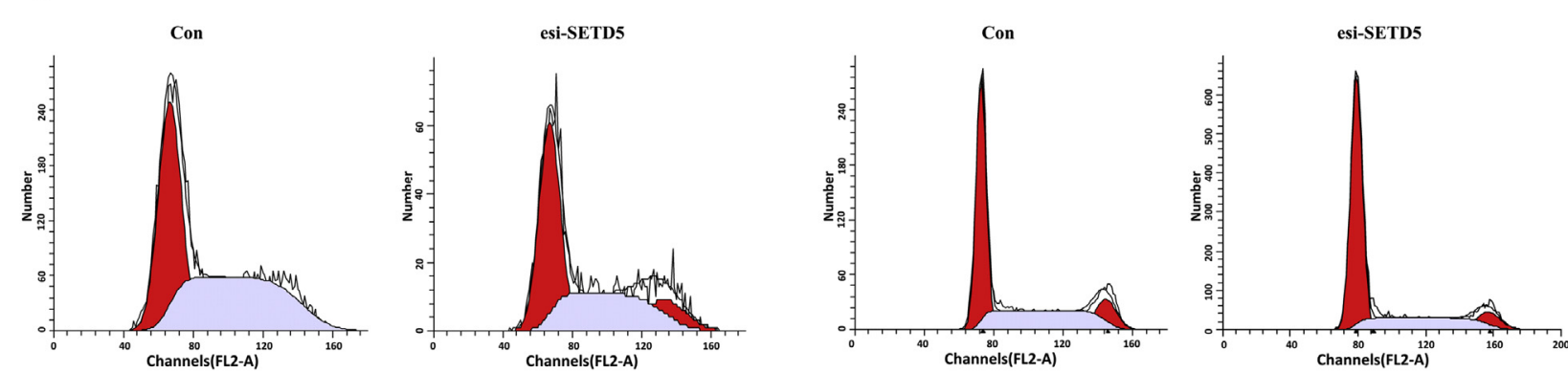

Figure 3 SETD5 positively regulates breast cancer (BC) cell proliferation. A: Effects of silencing SETD5 in MDA-MB-231 and MCF-7 cells evaluated by Western blot analysis. $\beta$-Actin served as control. B: Apoptosis-associated morphologic changes of BC cells analyzed using Hoechst 33258 fluorescent staining. C: Western blot assay showing the expression of apoptosis-related proteins in BC cells in the control (Con) group versus the SETD5 endoribonuclease-prepared short interfering RNA (esi-SETD5) group. D and E: Cell proliferation in the SETD5-knockdown group versus the control group based on carboxy-fluorescein succinimidyl ester (CFSE) staining (D) and colony formation assay (E). F: Representative histograms of the cell cycle distribution in two BC cell lines transfected with esi-SETD5 versus the control cells based on flow cytometry. ${ }^{* *} P<0.001$ versus controls. Scale bars $=100 \mu \mathrm{m}$ (B). Original magnification, $\times 200$ (B). Bax, Bcl2 associated X; Bcl-2, B-cell lymphoma 2; FITC, fluorescein isothiocyanate; PARP, poly ADP ribose polymerase.

involved in metastasis and angiogenesis are still unidentified. To determine the effects of SETD5 on angiogenesis, costaining for SETD5 and microvessel density marker $\mathrm{CD} 105^{27}$ was performed via IHC. The results revealed that CD105 was highly expressed in new capillary blood vessels near the cancer cells in the host (Supplemental Figure S3). Microvessel density was significantly higher in SETD5positive tissues of patients with $\mathrm{BC}(36.64 \pm 15.395)$ compared with that in SETD5-negative BC patient tissues $(30 \pm 13.172 ; P=0.039)$ (Figure 5A). The process of angiogenesis is mediated by several classic factors, including vascular endothelial growth factor (VEGF), and other endogenous nonclassic peptides, such as erythropoietin (EPO). ${ }^{28}$ Inhibition of SETD5 expression reduced the protein levels of VEGF and EPO (Figure 5B). Tube formation assays (Figure 5C) and vascular mimicry assays (Figure 5D) showed that SETD5 knockdown significantly inhibited tube formation, which was quantified as total branching length and intersection number, and confirmed the proangiogenic activity of SETD5.

\section{SETD5 Enhances BC Tumorigenesis through the AKT1 Signaling Pathway}

Many studies have shown that critical epigenetic modifiers are regulated by the PI3K/AKT signaling pathway and are involved in the oncogenicity of the PI3K cascade in cancers. ${ }^{29}$ To identify the potential mechanism of SETD5 in $\mathrm{BC}$, gene expression signatures of SETD5 high-expressing $\mathrm{BC}$ were investigated using GSEA and a public data set. Herein, 27 SETD5 high-expressing and 27 SETD5 lowexpressing $\mathrm{BC}$ groups were chosen from a sample pooled cohort of 54 patients with BC in the Gene Expression Omnibus database. GSEA showed that SETD5 expression levels were closely related to the PI3K/AKT signaling pathway (normalized enrichment score $=1.53$; false discovery rate $\mathrm{q}=0.19$ ) (Figure $6 \mathrm{~A}$ ). Tissue microarray analysis of BC tissues revealed a positive correlation between SETD5 expression and the expression of genes linked to PI3K/AKT signaling pathway activation, including $\mathrm{p} 85 \alpha$ $(P=0.004)$ and phosphorylated AKT (pAKT)-Ser473 


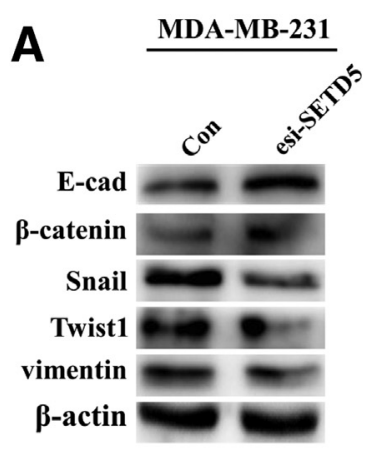

C

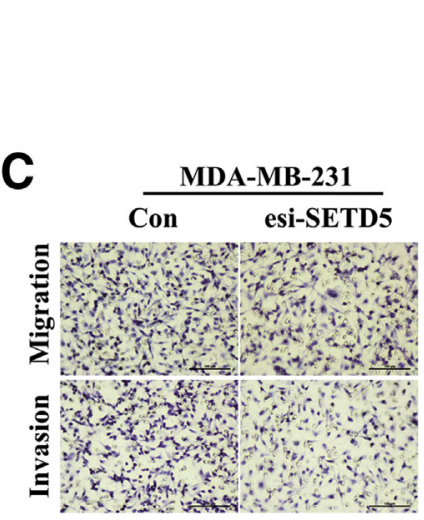

MCF-7

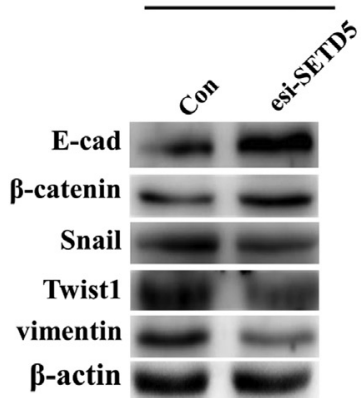

B

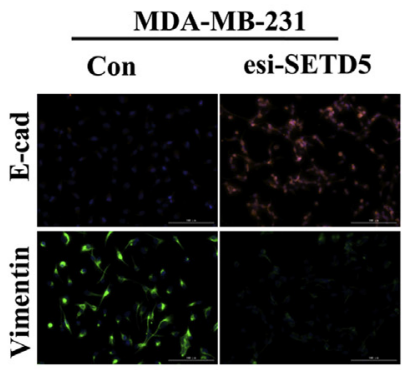

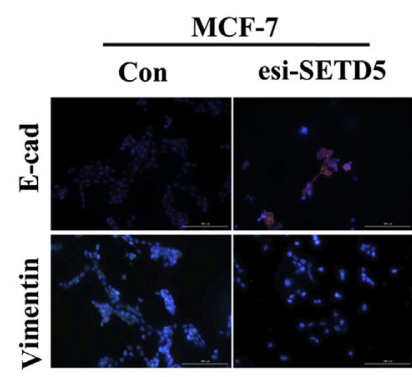
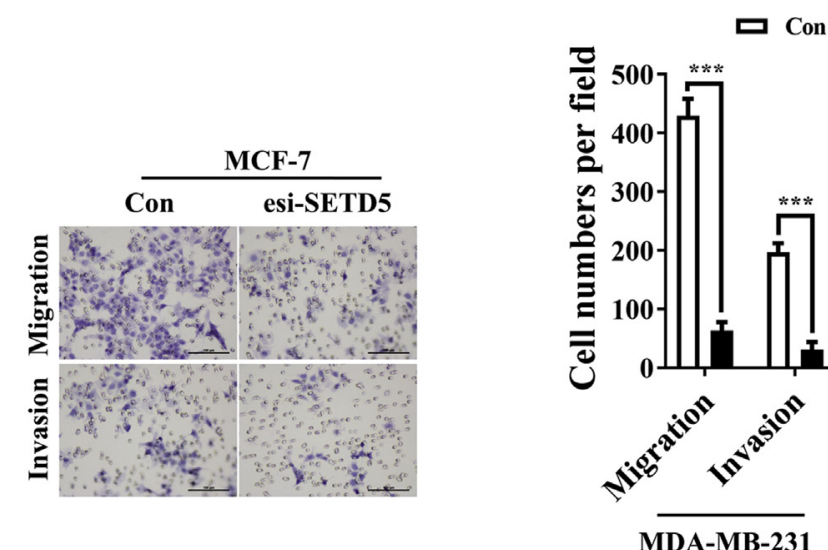

esi-SETD5

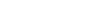

MDA-MB-231

Figure 4 SETD5 positively modulates epithelial-mesenchymal transition (EMT) in breast cancer (BC). A: Western blot analysis of EMT marker expression in BC cells and cells transfected with SETD5 endoribonuclease-prepared short interfering RNA (esi-SETD5). $\beta$-Actin was used as the loading control (Con). B: The expression of E-cadherin (E-cad) and vimentin was determined by immunofluorescence. C: MDA-MB-231 and MCF-7 cell migration and invasion ability analyzed using transwell and Matrigel invasion assays after being transfected with esi-SETD5. The invading cells were stained and counted. ${ }^{*} P<0.05,{ }^{*} P<0.01$, and ${ }^{* * *} P<0.001$ versus controls. Scale bars $=100 \mu \mathrm{m}$ (B and $\left.\mathbf{C}\right)$. Original magnification, $\times 200$ (B and $\left.\mathbf{C}\right)$.

$(P=0.027)$ (Supplemental Figure S4, A-D, and Supplemental Table S1), which confirmed the GSEA results. Furthermore, when MCF-7 and MDA-MB-231 cells were transfected with esi-SETD5 or treated with inhibitors for PI3K (LY294002, $10 \mu \mathrm{mol} / \mathrm{L}$ ) or AKT1 (perifosine, 10 $\mu \mathrm{mol} / \mathrm{L}$ ), the upstream and downstream relationships among PI3K, AKT1, and SETD5 were confirmed. LY294002 and perifosine decreased AKT1 phosphorylation at pAKTThr308 and pAKT-Ser473, respectively. The expression of SETD5 was not significantly down-regulated in the cells when treated with LY294002 or perifosine. However, silencing of SETD5 had a marked effect on AKT1 phosphorylation at pAKT-Thr308 and pAKT-Ser473 (Figure 6B). SETD5 was clearly pulled down by an antiAKT1 antibody in BC cells during co-IP, which indicated close binding between SETD5 and AKT1 (Figure 6C). Moreover, immunofluorescence was used to evaluate the correlation between SETD5 expression and AKT1. The results showed that SETD5 was colocated with AKT1 phosphorylation at pAKT-Thr308 and pAKT-Ser473 in BC patient tissues (Figure 6D). The positive relationship between the expression of SETD5 and AKT1 mRNA levels was further analyzed by Spearman correlation analysis of Gene Expression Profiling Interactive Analysis $(R=0.25$; $P<0.001$ ) (Supplemental Figure S4E). This points towards SETD5 working as an upstream signal of AKT1 and regulating AKT1 phosphorylation in $\mathrm{BC}$ cells as a possible mechanism.

\section{Experiments in Vivo}

To further confirm whether SETD5 affects the proliferation of tumor cells, SETD5-depleted MDA-MB-231 cells were injected (subcutaneously) into the right flank of 5-week-old female BALB/c nude mice. Inhibition of SETD5 expression significantly inhibited tumor growth (Figure 7A). Inhibition of SETD5 significantly reduced the scores of the cancer cells stained by Ki-67 (a typical biomarker of cell proliferation), vimentin, VEGF, and EPO, and increased the expression of cleaved caspase-3 and E-cadherin in xenograft tumor tissues, as indicated by IHC (Figure 7, B and C). Collectively, these observations suggest that SETD5 can induce the growth of BC tumors, supporting the hypothesis that SETD5-targeted endoribonuclease-prepared short interfering RNA may function as a suppressor of $\mathrm{BC}$ tumors.

In vivo pulmonary metastasis experiments were subsequently performed to further confirm the role of SETD5 in BC cell micrometastases. Metastatic nodules of tumor xenografts were counted in lung sections of the mice 4 weeks after tail vein injection. Inhibiting the expression of SETD5 was found to markedly reduce the numbers of metastatic lung nodules (Figure 7D). Histologic analysis confirmed the 
A

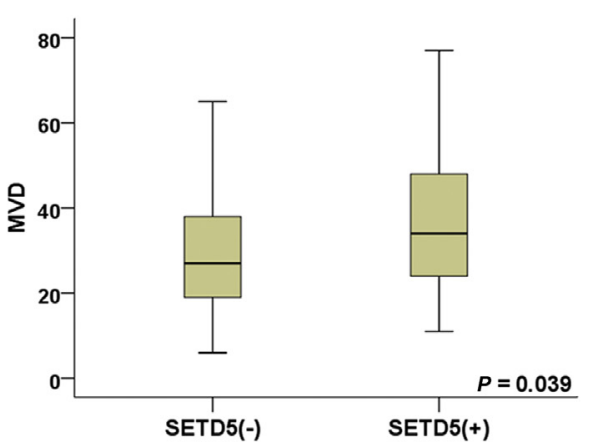

B
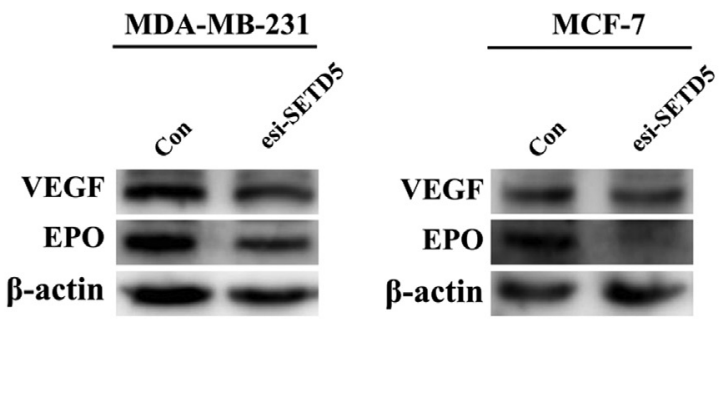

\section{C}

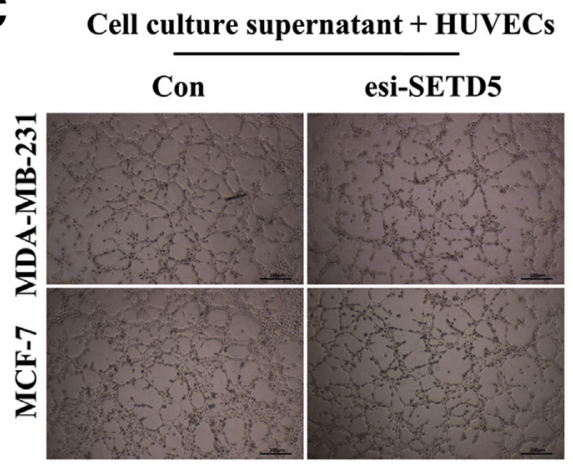

D

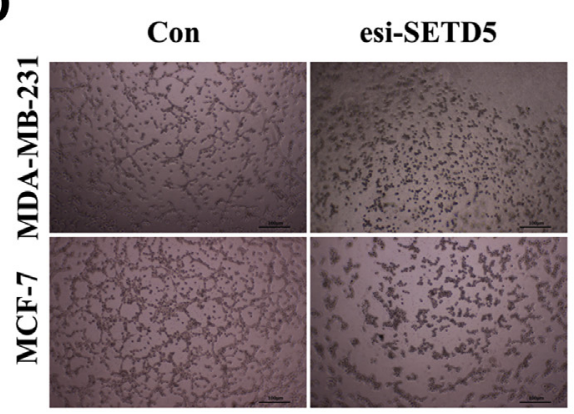

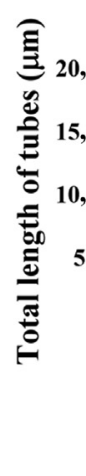

口 Con

esi-SETDS

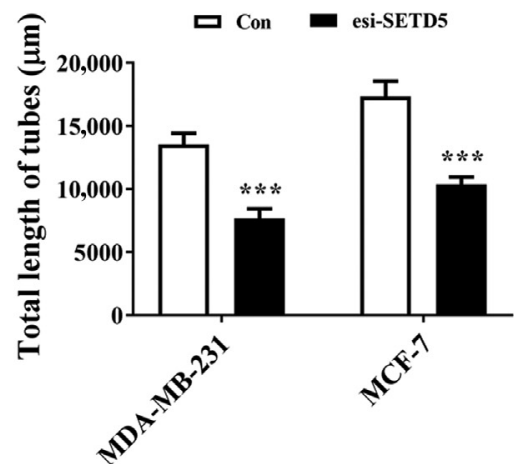

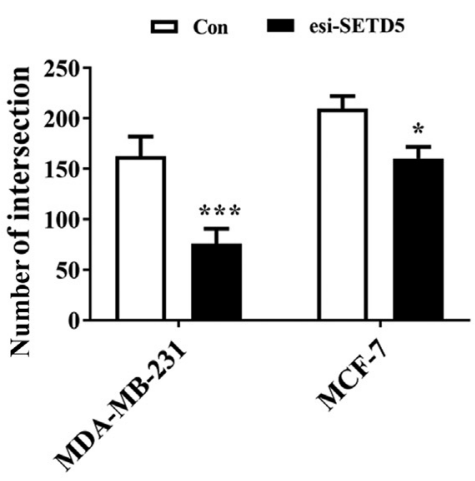

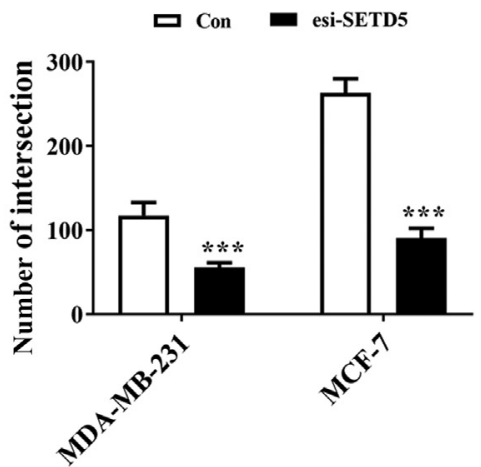

Figure 5 Proangiogenic role of SETD5 in breast cancer (BC). A: Association between SETD5 and CD105 in BC tissues. B: Western blot analysis of vascular endothelial growth factor (VEGF) and erythropoietin (EP0) protein expression in normal MCF-7 and MDA-MB-231 cells and cells transfected with SETD5 endoribonuclease-prepared short interfering RNA (esi-SETD5). C and D: Tube formation assays (C) and vasculogenic mimicry assays (D) performed to analyze the effect of esi-SETD5 on tumor angiogenesis. Quantitation of total branching length of tubes and numbers of intersections performed using ImageJ software version 1.52 with the angiogenesis plugin. ${ }^{*} P<0.05,{ }^{* *} P<0.001$ versus controls (Con). Scale bars $=100 \mu \mathrm{m}$ (C and D). Original magnification, $\times 100$ (C and D). HUVEC, human umbilical vein endothelial cell; MVD, microvessel density.

presence of $\mathrm{BC}$ metastases in the lungs of the mice, as demonstrated by the photomicrographs of hematoxylin and eosin-stained specimens (Figure 7D). Collectively, these data clearly demonstrate a vital role of SETD5 in the occurrence of pulmonary metastasis in BC.

\section{Discussion}

SETD5 has H3K36 methyltransferase activity, which is crucial in the elongation and processing of RNA, as well as correct gene transcription. ${ }^{30}$ Alteration in promoter-proximal pausing of the polymerase usually impairs this process, thus leading to several pathologic disorders, such as developmental syndromes and cancer. ${ }^{31,32}$ Although the up-regulation of SETD5 in BC has been previously reported, ${ }^{15}$ the fact that SETD5 is related to poor disease outcomes in patients with $\mathrm{BC}$ and the mechanisms underlying its involvement in tumor progression have not been elucidated. The current study involves a comprehensive investigation of SETD5 with respect to tumor progression, which eventually results in a poor prognosis for patients with BC. First, the expression of SETD5 was shown to be increased in $\mathrm{BC}$ tissues and be positively associated with lymph nodes metastasis, advanced clinical stages, and patient OS. Additionally, SETD5 was determined to be an independent prognostic factor for patients with BC. Second, SETD5 was shown to be 
A

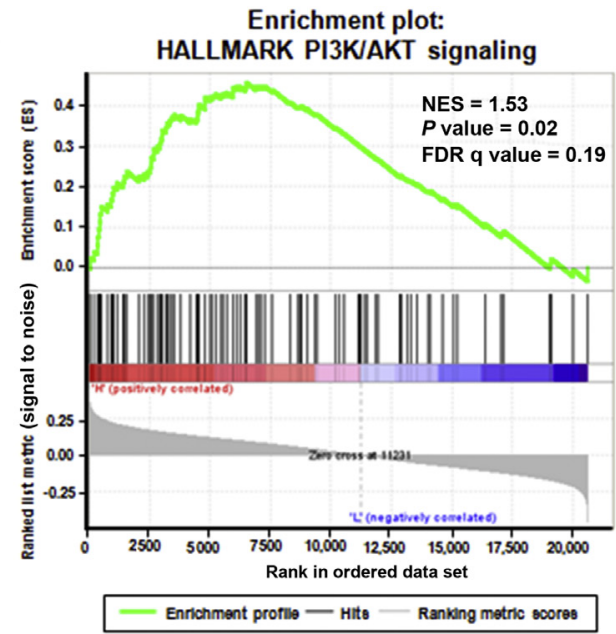

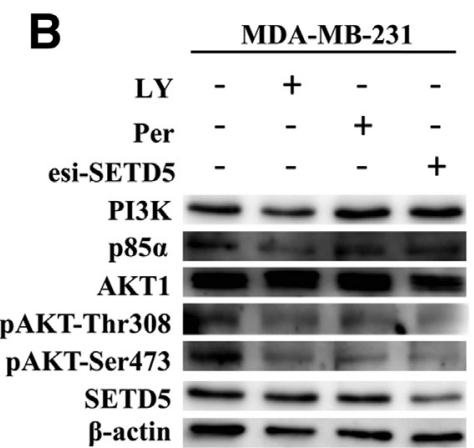

C

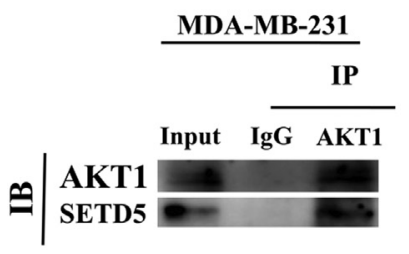

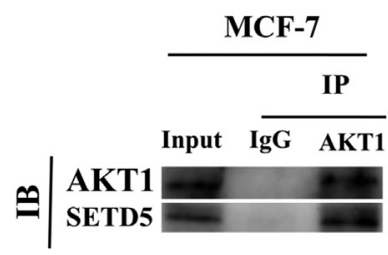

DAPI

SETD5

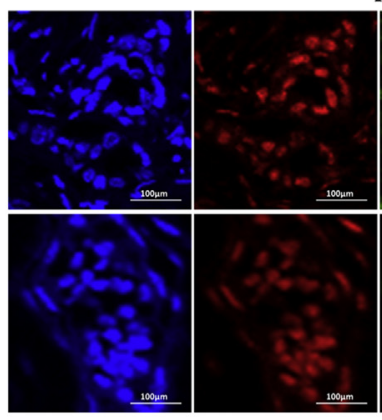

DAPI

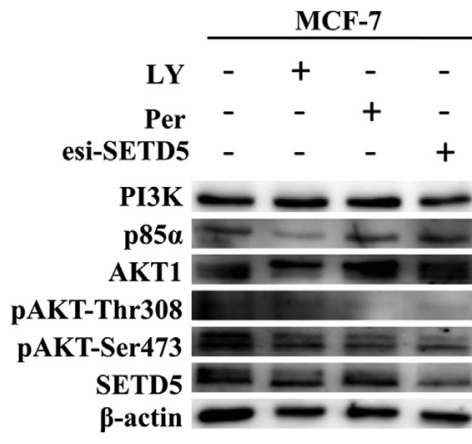

AKT-Thr308 Merge

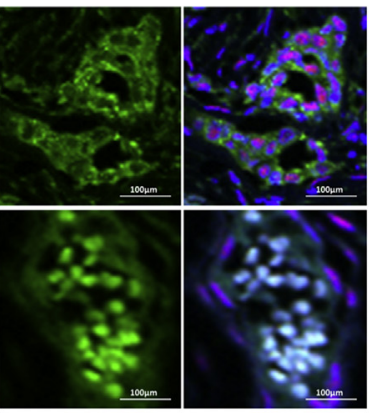

pAKT-Ser473 Merge

Figure 6 SETD5 interacts with AKT1 in breast cancer (BC). A: Gene Set Enrichment Analysis results for phosphoinositide 3-kinase (PI3K)/AKT in SETD5 high-expressing BC. B: Western blot analysis of PI3K, p85 $\alpha$, AKT1, phosphorylated AKT (pAKT)-Thr308, pAKT-Ser473, and SETD5 in MDA-MB-231 and MCF-7 cells after treatment with the PI3K inhibitor LY294002 (LY) or AKT inhibitor perifosine (Per) or transfection with SETD5 endoribonuclease-prepared short interfering RNA (esi-SETD5). C: SETD5 and AKT1 protein interactions in MDA-MB-231 and MCF7 cells were analyzed by co-immunoprecipitation (IP). D: Immunofluorescence analysis of the colocalization of SETD5 and AKT1 phosphorylation at pAKT-Thr308 and pAKT-Ser473 in BC tissues. Scale bars $=100 \mu \mathrm{m}$ (D). Original magnification, $\times 200$ (D). FDR, false discovery rate; IB, immunoblot; NES, normalized enrichment score.

an upstream signal of AKT1 in BC cells. Third, inhibition of SETD5 suppressed the tumor growth and metastasis of BC cells in vivo and in vitro. Collectively, these data demonstrated that SETD5 promotes tumor progression via AKT1 signaling, ultimately leading to a poor prognosis for patients with BC.

The expression of SETD7 of the SETD methyltransferase family of genes is positively associated with metastasis, recurrence, and poor prognosis of hepatocellular carcinoma. ${ }^{3}$ Consistent with the report by Chen et $\mathrm{al},{ }^{33}$ the current study found that SETD5 had high expression in BC tissues and positively correlated with patient age, lymph node metastasis, and clinical stage. Survival analyses showed that expression of SETD5 was significantly related to a poorer prognosis. Cox regression analysis indicated that the expression of SETD5 was an independent prognostic factor for patients with BC. While the collective data support the potential value of SETD5 as a novel prognostic marker for BC, the role of SETD5 in BC tumorigenesis is not fully understood.

Studies have shown that embryonic stem cells lacking SETD5 expression show high apoptosis rate, poor proliferation, defective cell cycle progression, weakened ability to differentiate to cardiomyocytes, and poor gene expression. ${ }^{20,34}$ In the current study, SETD5-deficient BC cells were found to induce tumor cell apoptosis and to inhibit tumor cell proliferation in vitro. A xenograft mouse model further confirmed that knockdown of SETD5 inhibited tumor growth. Also, in vivo experiments further confirmed the biological function of SETD5 regarding cancer cell apoptosis and proliferation. Thus, it is conceivable that SETD5 expression decreases apoptosis and promotes proliferation of $\mathrm{BC}$ cells.

$\mathrm{Yu}$ et $\mathrm{al}^{35}$ have shown that SETD5 may promote the invasive and migration abilities of non-small-cell lung cancer by promoting Snail expression, while suppressing zonula occludens protein 1 expression. It will be interesting to determine whether SETD5 regulates the expression of molecules related to the EMT in BC. Consistent with this, the current study show that SETD5 knockdown influenced the expression of EMT-related genes in both BC cells and xenograft tumor tissues. The most important clinical significance of the EMT process is its influence on BC cell invasion and metastasis. Inhibiting the expression of SETD5 was found to lead to decreased cell migration and invasion 

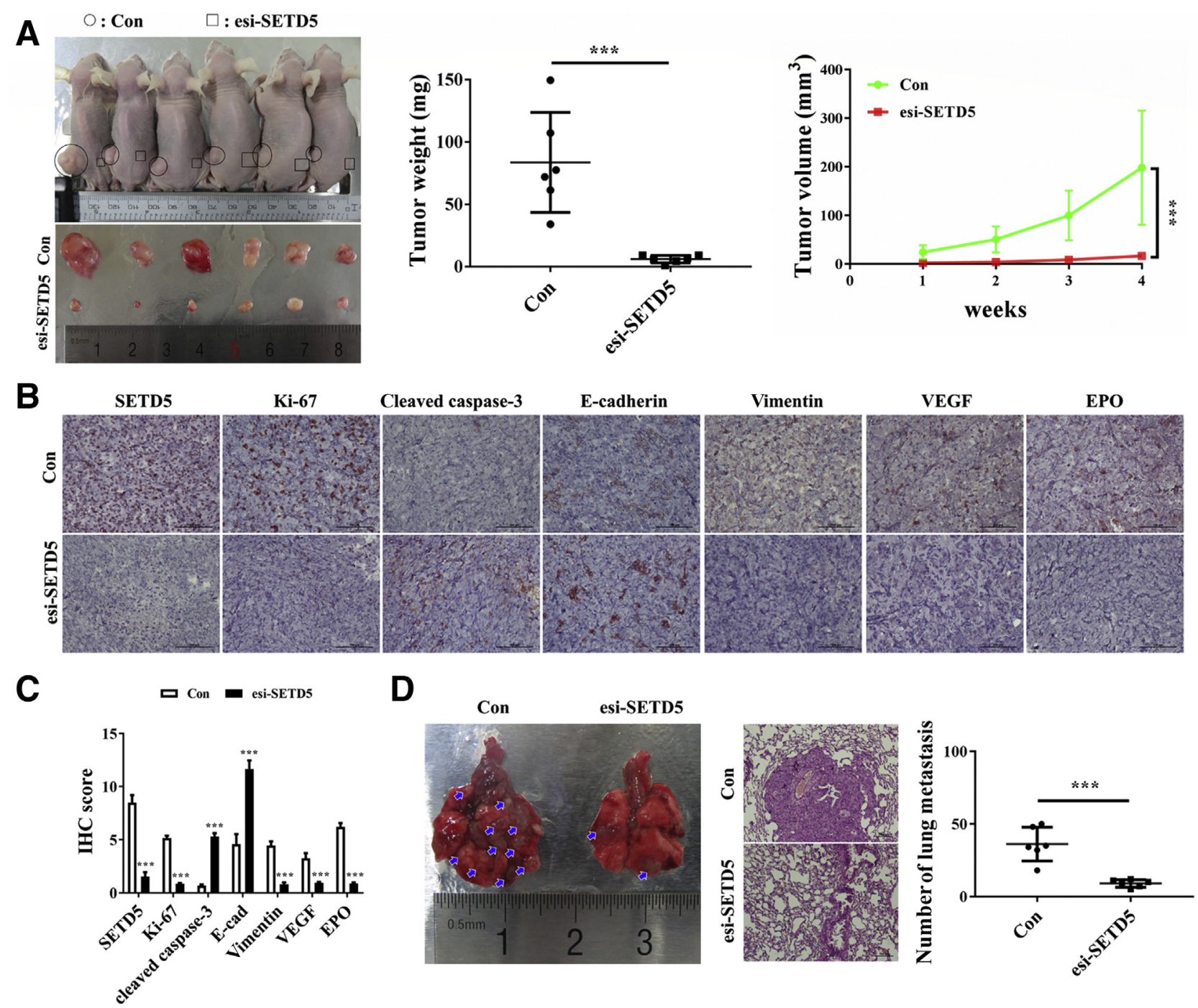

Figure 7 The anticancer growth effect of SETD5 endoribonuclease-prepared short interfering RNA (esi-SETD5) in vivo. A: Mice with tumors formed by subcutaneously injected normal MDA-MB-231 cells (black circles) and MDA-MB-231 cells transfected with esi-SETD5 (black squares). Tumor volumes and tumor weights of each group at the indicated time after injection are shown. B and C: Immunohistochemistry (IHC) staining and scores of SETD5, Ki-67, cleaved caspase-3, E-cadherin (E-cad), vimentin, vascular endothelial growth factor (VEGF), and erythropoietin (EP0) in xenograft tumors. D: MDA-MB-231 cells and MDA-MB-231 cells transfected with esi-SETD5 were injected via tail-vein injection into the mice. Representative micrographs of hematoxylin and eosin-stained metastatic nodules. Metastatic nodules (blue arrows) in the lungs were counted. ${ }^{* *} P<0.001$ versus controls (Con). Scale bars $=100 \mu \mathrm{m}$ (B and D). Original magnification: $\times 200$ (B); $\times 100$ (D).

in vitro. More important, SETD5 knockdown inhibited the metastasis of BC cells to the lungs in vivo. Taken together, it is conceivable that SETD5 expression may lead to EMT program and promote lung micrometastases of $\mathrm{BC}$ cells.

Tumor angiogenesis refers to the formation of new blood vessels to serve the tumor tissues and is used as a therapeutic target in cancer treatment. Notably, the process is vital in the proliferation and metastasis of cancer cells. ${ }^{36,37}$ Here we used a series of experiments to explore the role of SETD5 in tumor angiogenesis, which has never been reported before. In the current study, IHC of specimens from patients with BC revealed that SETD5, along with CD105, was markedly upregulated in cancer tissues in comparison to the adjacent tissues. Additional studies showed that SETD5 influenced total branching length and the intersection number of tumor blood vessels and promoted angiogenesis and VEGF and EPO expression in BC cells. In addition, silencing SETD5 was demonstrated to down-regulate the expression of VEGF and $\mathrm{EPO}$ in $\mathrm{BC}$ xenograft tumor tissues. These findings may have significant implications for treating $\mathrm{BC}$ angiogenesis via targeting SETD5, which, in turn, may further suppress tumor proliferation, invasion, and metastasis. However, the molecular mechanisms by which SETD5 affects angiogenesis and promotes $\mathrm{BC}$ cell proliferation, invasion, and metastasis require further investigation.

Development of anticancer drug resistance has been associated with the activation of the PI3K/AKT pathway. AKT activation leads to the phosphorylation of many substrates, as well as downstream effectors, including members of the caspase family and cell cycle protein family, which 
facilitate cell survival, proliferation, metastasis, and chemoresistance. ${ }^{38-40}$ Thus, it is logical to investigate the association between SETD5 and AKT in BC. (Figure 6), GSEA analysis, IHC staining, and co-IP assays clearly indicated that SETD5 expression was closely related to AKT1 signaling in BC. Furthermore, silencing of SETD5 resulted in decreased AKT1 phosphorylation at pAKTThr308 and pAKT-Ser473. These observations suggest that SETD5 signaling functions as an upstream signal of AKT1 and is crucial for the targeted activation of AKT1. The current findings broaden understanding of SETD5-regulated BC progression and demonstrate that this regulation was primarily through the AKT1 pathway.

In conclusion, SETD5 may act as a potential prognostic biomarker for patients with BC. The current study reports a novel role for SETD5 in promoting tumor progression by activating the AKT1 pathway in $\mathrm{BC}$ cells, promoting $\mathrm{BC}$ cell growth and motility in vitro and facilitating tumor growth and metastasis in vivo.

\section{Acknowledgments}

We thank Editage (Beijing, China) for English-language editing.

\section{Supplemental Data}

Supplemental material for this article can be found at http://doi.org/10.1016/j.ajpath.2020.10.005.

\section{References}

1. Bray F, Ferlay J, Soerjomataram I, Siegel RL, Torre LA, Jemal A: Global cancer statistics 2018: GLOBOCAN estimates of incidence and mortality worldwide for 36 cancers in 185 countries. CA Cancer J Clin 2018, 68:394-424

2. DeSantis CE, Ma J, Gaudet MM, Newman LA, Miller KD, Goding Sauer A, Jemal A, Siegel RL: Breast cancer statistics, 2019. CA Cancer J Clin 2019, 69:438-451

3. Duffy MJ, O'Donovan N, Crown J: Use of molecular markers for predicting therapy response in cancer patients. Cancer Treat Rev 2011, 37:151-159

4. Revathidevi S, Munirajan AK: Akt in cancer: mediator and more Semin Cancer Biol 2019, 59:80-91

5. Datta SR, Brunet A, Greenberg ME: Cellular survival: a play in three akts. Genes Dev 1999, 13:2905-2927

6. Alessi DR, Andjelkovic M, Caudwell B, Cron P, Morrice N, Cohen P, Hemmings BA: Mechanism of activation of protein kinase B by insulin and IGF-1. EMBO 1996, 15:6541-6551

7. Altomare DA, Testa JR: Perturbations of the AKT signaling pathway in human cancer. Oncogene 2005, 24:7455-7464

8. Nakakido M, Deng Z, Suzuki T, Dohmae N, Nakamura Y, Hamamoto R: Dysregulation of AKT pathway by SMYD2-mediated lysine methylation on PTEN. Neoplasia 2015, 17:367-373

9. Xiao B, Wilson JR, Gamblin SJ: SET domains and histone methylation. Curr Opin Struct Biol 2003, 13:699-705

10. Dillon SC, Zhang X, Trievel RC, Cheng X: The SET-domain protein superfamily: protein lysine methyltransferases. Genome Biol 2005, 6: $227-236$
11. Grozeva D, Carss K, Spasic-Boskovic O, Parker MJ, Archer H, Firth HV, Park SM, Canham N, Holder SE, Wilson M, Hackett A, Field M, Floyd JAB, Hurles M, Lucy Raymond F: De novo loss-offunction mutations in SETD5, encoding a methyltransferase in a 3p25 microdeletion syndrome critical region, cause intellectual disability Am J Hum Genet 2014, 94:618-624

12. Kuechler A, Zink AM, Wieland T, Lüdecke HJ, Cremer K, Salviati L, Magini P, Najafi K, Zweier C, Czeschik JC, Aretz S, Endele S, Tamburrino F, Pinato C, Clementi M, Gundlach J, Maylahn C, Mazzanti L, Wohlleber E, Schwarzmayr T, Kariminejad R, Schlessinger A, Wieczorek D, Strom TM, Novarino G, Engels H: Loss-of-function variants of SETD5 cause intellectual disability and the core phenotype of microdeletion 3p25.3 syndrome. Eur J Hum Genet 2015, 23:753-760

13. Parenti I, Teresa-Rodrigo ME, Pozojevic J, Ruiz Gil S, Bader I, Braunholz D, Bramswig NC, Gervasini C, Larizza L, Pfeiffer L, Ozkinay F, Ramos F, Reiz B, Rittinger O, Strom TM, Watrin E, Wendt K, Wieczorek D, Wollnik B, Baquero-Montoya C, Pié J, Deardorff MA, Gillessen-Kaesbach G, Kaiser FJ: Mutations in chromatin regulators functionally link Cornelia de Lange syndrome and clinically overlapping phenotypes. Hum Genet 2017, 136:307-320

14. Szczałuba K, Brzezinska M, Kot J, Rydzanicz M, Walczak A, Stawiński P, Werner B, Płoski R: SETD5 loss-of-function mutation as a likely cause of a familial syndromic intellectual disability with variable phenotypic expression. Am J Med Genet A 2016, 170:2322-2327

15. Liu L, Kimball S, Liu H, Holowatyj A, Yang ZQ: Genetic alterations of histone lysine methyltransferases and their significance in breast cancer. Oncotarget 2015, 6:2466-2482

16. Yang ZT, Yeo SY, Yin YX, Lin ZH, Lee HM, Xuan YH, Cui Y, Kim SH: Tenascin-C, a prognostic determinant of esophageal squamous cell carcinoma. PLoS One 2016, 11:1-14

17. Yang ZT, Cui Y, Ni WD, Kim SY, Xuan YH: Gli1, a potential regulator of esophageal cancer stem cell, is identified as an independent adverse prognostic factor in esophageal squamous cell carcinoma. J Cancer Res Clin Oncol 2017, 143:243-254

18. Yang ZT, Zhang CY, Qi WB, Cui Y, Xuan YH: GLI1 promotes cancer stemness through intracellular signaling pathway PI3K/Akt/NFkB in colorectal adenocarcinoma. Exp Cell Res 2018, 373:145-154

19. Committee for the Update of the Guide for the Care and Use of Laboratory Animals: National Research Council: Guide for the Care and Use of Laboratory Animals: Eighth Edition. Washington, DC, National Academies Press, 2011

20. Osipovich AB, Gangula R, Vianna PG, Magnuson MA: Setd5 is essential for mammalian development and the co-transcriptional regulation of histone acetylation. Development 2016, 143:4595-4607

21. Kovaleva OV, Samoilova DV, Shitova MS, Gratchev A: Tumor associated macrophages in kidney cancer. Anal Cell Pathol Cell Oncol 2016, 2016:9307549-9307554

22. Prall F: Tumour budding in colorectal carcinoma. Histopathology 2007, 50:151-162

23. Mitrovic B, Schaeffer DF, Riddell RH, Kirsch R: Tumor budding in colorectal carcinoma: time to take notice. Mod Pathol 2012, 25: $1315-1325$

24. Zlobec I, Lugli A: Epithelial mesenchymal transition and tumor budding in aggressive colorectal cancer: tumor budding as oncotarget. Oncotarget 2010, 1:651-661

25. Mallini P, Lennard T, Kirby J, Meeson A: Epithelial-to-mesenchymal transition: what is the impact on breast cancer stem cells and drug resistance. Cancer Treat Rev 2014, 40:341-348

26. Lee JM, Dedhar S, Kalluri R, Thompson EW: The epithelial-mesenchymal transition: new insights in signaling, development, and disease. J Cell Biol 2006, 172:973-981

27. Tanaka F, Otake Y, Yanagihara K, Kawano Y, Miyahara R, Li M, Yamada T, Hanaoka N, Inui K, Wada H: Evaluation of angiogenesis in non-small cell lung cancer: comparison between anti-CD34 antibody and anti-CD105 antibody. Clin Cancer Res 2001, 7:3410-3415 
28. Ribatti D, Tamma R: Hematopoietic growth factors and tumor angiogenesis. Cancer Lett 2019, 440-441:47-53

29. Yang Q, Jiang W, Hou P: Emerging role of PI3K/AKT in tumorrelated epigenetic regulation. Semin Cancer Biol 2019, 59:112-124

30. Sessa A, Fagnocchi L, Mastrototaro G, Massimino L, Zaghi M, Indrigo M, Cattaneo S, Martini D, Gabellini C, Pucci C, Fasciani A, Belli R, Taverna S, Andreazzoli M, Zippo A, Broccoli V: SETD5 regulates chromatin methylation state and preserves global transcriptional fidelity during brain development and neuronal wiring. Neuron 2019, 104:271-289

31. Mayer A, Landry HM, Churchman LS: Pause \& go: from the discovery of RNA polymerase pausing to its functional implications. Curr Opin Cell Biol 2017, 46:72-80

32. Jonkers I, Lis JT: Getting up to speed with transcription elongation by RNA polymerase II. Nat Rev Mol Cell Biol 2015, 16:167-177

33. Chen Y, Yang S, Hu J, Yu C, He M, Cai Z: Increased expression of SETD7 promotes cell proliferation by regulating cell cycle and indicates poor prognosis in hepatocellular carcinoma. PLoS One 2016, $11: 1-14$

34. Blagosklonny MV: Target for cancer therapy: proliferating cells or stem cells. Leukemia 2006, 20:385-391

35. Yu H, Sun J, Zhao C, Wang H, Liu Y, Xiong J, Chang J, Wang M, Wang W, Ye D, Zhou H, Yu T: SET domain containing protein 5
(SETD5) enhances tumor cell invasion and is associated with a poor prognosis in non-small cell lung cancer patients. BMC Cancer 2019, 19:736-745

36. Lin QH, Qu W, Xu J, Feng F, He MF: 1-Methoxycarbony- $\beta$-carboline from Picrasma quassioides exerts anti-angiogenic properties in HUVECs in vitro and zebrafish embryos in vivo. Chin J Nat Med 2018, 16:599-609

37. Luan X, Gao YG, Guan YY, Xu JR, Lu Q, Zhao M, Liu YR, Liu HJ, Fang C, Chen HZ: Platycodin D inhibits tumor growth by antiangiogenic activity via blocking VEGFR2-mediated signaling pathway. Toxicol Appl Pharmacol 2014, 281:118-124

38. Cheng JQ, Lindsley CW, Cheng GZ, Yang H, Nicosia SV: The Akt/PKB pathway: molecular target for cancer drug discovery. Oncogene 2005, 24:7482-7492

39. Andre F, Nahta R, Conforti R, Boulet T, Aziz M, Yuan LXH, Meslin F, Spielmann M, Tomasic G, Pusztai L, Hortobagyi GN, Michiels S, Delaloge S, Esteva FJ: Expression patterns and predictive value of phosphorylated AKT in early-stage breast cancer. Ann Oncol 2008, 19:315-320

40. Liang K, Lu Y, Li X, Zeng X, Glazer RI, Mills GB, Fan Z: Differential roles of phosphoinositide-dependent protein kinase-1 and Akt1 expression and phosphorylation in breast cancer cell resistance to paclitaxel, doxorubicin, and gemcitabine. Mol Pharmacol 2006, 70:1045-1052 